\title{
Synthesis of Antennas for Field and Polarization Control
}

\author{
Leandro P. S. Pereira, Marco A. B. Terada \\ Antenna Group, Electrical Engineering Department, University of Brasilia, Brasilia, Brazil \\ Email:terada@unb.br
}

How to cite this paper: Pereira, L.P.S. and Terada, M.A.B. (2017) Synthesis of Antennas for Field and Polarization Control. Journal of Electromagnetic Analysis and Applications, 9, 97-112. https://doi.org/10.4236/jemaa.2017.97009

Received: May 23, 2017

Accepted: July 16, 2017

Published: July 19, 2017

Copyright $\odot 2017$ by authors and Scientific Research Publishing Inc. This work is licensed under the Creative Commons Attribution International License (CC BY 4.0).

http://creativecommons.org/licenses/by/4.0/ (c) (i) Open Access

\begin{abstract}
This article presents a procedure for electromagnetic field and polarization control with antennas. The concept previously introduced by the authors for spatially distributed three-dimensional electromagnetic polarization (as time varies) is discussed and extended also to include non-ideal antennas and the control of electromagnetic field distributions (at a given instant of time). These polarizations and fields are herein referred to as " $3 \mathrm{D}$ ", although time is also inherent to them. Even that the main objective is to introduce a mathematically/numerically consistent synthesis technique for controlling the 3D electromagnetic fields and polarizations, an effort is made to present and discuss possible applications, including but not limited to torus-knotted distributions and spatial multiplexing for transmission of information in wireless digital communication systems.
\end{abstract}

\section{Keywords}

Antennas, Electromagnetic Theory, Electric Fields, Electromagnetic Polarization, Three-Dimensional Polarization and Orthogonality, Spatial Multiplexing, Transmission of Information, Cellular Communication Systems, Torus-Knots

\section{Introduction}

The increasing usage of electromagnetic polarization in telecommunication systems is well known and documented, ranging from applications such as diversity and frequency reuse to electromagnetic compatibility and future $4 \mathrm{k} / 8 \mathrm{k}$ resolution Digital Television Broadcasting [1]-[6]. The polarization of a wave is defined by the path described by the electric field vector at a fixed point in space as time varies, and the polarization of an antenna is defined as the polarization of the wave it radiates when operating in transmitting mode [6]. The possibility of 
generating 3D polarizations may lead to enhancements in the existing telecommunication systems or even the development of new configurations and applications.

This work presents a way of generating 3D polarizations and field distributions, where the electric field intensity vector can be oriented in any direction in space. Therefore, it is possible to generate $3 \mathrm{D}$ polarizations and/or fields with different shapes, as time varies, such as cylinders, spheres, and others. In addition, the orthogonality between 3D polarizations is also discussed. Antennas naturally generate a polarization that is orthogonal to the desired one, normally referred to as cross polarization, but the idea here is to generate the orthogonal polarizations in a controlled manner, similarly to the use of orthogonal polarizations in frequency reuse systems. Cross polarization is normally undesired, with the exception of the application introduced in [7].

It was previously demonstrated analytically and numerically by the authors in [8] that 3D polarizations can be generated at an observation point inside a $3 \mathrm{D}$ antenna array, where the elements are facing inwards. Volumetric control of the intensity of electric fields and sound has been previously published [9] [10] [11] [12], but as far as the authors know, generation and application of 3D polarizations and fields have not yet been investigated in the way herein presented.

It was demonstrated in [8] that ideal antennas can generate 3D polarizations, and here the concept is extended for pyramidal horn antennas, as well as spatially distributed electromagnetic fields. A new method introduced by the authors for optimum design of these antennas was also employed in order to ensure greater gain stability with respect to manufacturing variations [13].

A brief summary of the concept discussed in [8] is presented for completeness, also illustrating different scenarios than the ones covered in [8], and it is shown that antenna arrays can be used for spatial multiplexing in wireless communication systems by controlling the fields in $3 \mathrm{D}$ space, in order to reduce interference among users. This might be critical in the actual trending of the evolution of cellular systems towards nano and phantom cells [14]. Another possible application for the field and polarization control herein discussed is the generation of torus-knotted polarizations and fields, where the latter can be encountered in nature around planets, stars and other celestial objects, as indicated historically in Radioastronomy as well as possibly to be further studied with state-of-the-art Radiotelescopes [15], and may be of importance for plasma confinement and nuclear magnetic resonance devices [16] [17].

\section{Three-Dimensional Polarization with Ideal Dipoles (Near-Field) and Orthogonality}

The antenna array shown in Figure 1 is used to generate 3D polarizations at the observation point $O$, and was derived from the antenna array configurations introduced by one the authors in [9] [10]. All the computer simulations employed the computer package MathCad [18]. Note that with this configuration, each 


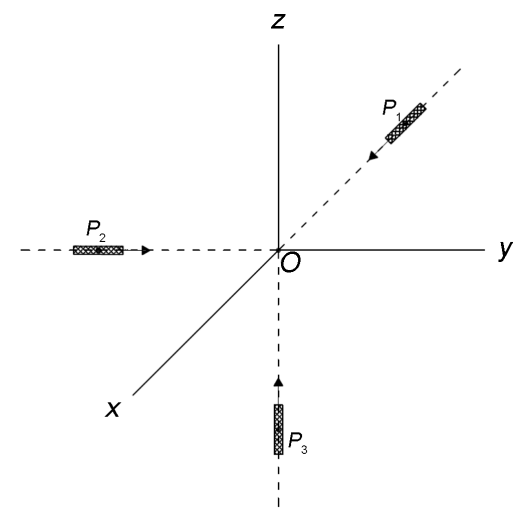

Figure 1. Array of 3 ideal dipole antennas in free space. Although not discussed in this work, further consideration can also be given to array configurations of moving elements, including ones in known paths.

antenna produces fields only in the radial direction. This was done to simplify the equations discussed in [8], even though it is possible to change the configuration for any desired direction. If the amplitude and phase of the antennas are constant, the resulting fields obtained by superposition (it is assumed a linear, homogenous, time invariant medium) normally lead to an elliptical polarization. However, by controlling the variations of the amplitude and/or phase of the elements it is possible to rotate this ellipse with time, and hence yielding a $3 \mathrm{D}$ polarization.

The electric field generated by one antenna is frequency dependent, however the figure formed by the fields generated in the array of ideal dipole are frequency independent if the same frequency is used for the all antennas. This occurs because the proportionality between the electric fields generated by individual antennas is kept constant when the frequency is changed. Thus, for illustration purposes, without loss of generality, we use $f=100 \mathrm{MHz}, I=1 \mathrm{~mA}, h=$ $\lambda / 100$ (the length of all 3 antennas placed at a distance equal to $\lambda$ from point $O$ ). It is important to note that for the scenarios considered in this study there is no propagation, so the $3 \mathrm{D}$ polarization needs to be visualized as the polarization of the resulting electric field, not the wave. Alternate configurations that allow the 3D polarization to move within a desired path are currently being investigated (by determining different sets of feed currents for each desired location along the trajectory, for example), but the theory does not lose its importance nor generality as herein presented. By varying continuously the phase of the ideal dipole $P_{2}$ in Figure 1, i.e. $\psi_{2}$ in the interval 0 to $2 \pi$, with $\psi_{1}=\psi_{3}=0^{\circ}$ respectively for the other two dipoles, the resulting polarization is shown in Figure 2. In general, 3 components (basis) of electric fields are necessary in order to generate a $3 \mathrm{D}$ polarization. In the case of the components being orthogonal, they form an orthogonal base; the reader is referred to [8] for further details.

\section{Three-Dimensional Polarization with Pyramidal Horn Antennas (Far-Field)}

In order to show that it is also possible to generate $3 \mathrm{D}$ polarizations with anten- 
nas other than the ideal dipoles, we employed the array of pyramidal horns shown in Figure 3. The geometry of each horn is depicted in Figure 4.

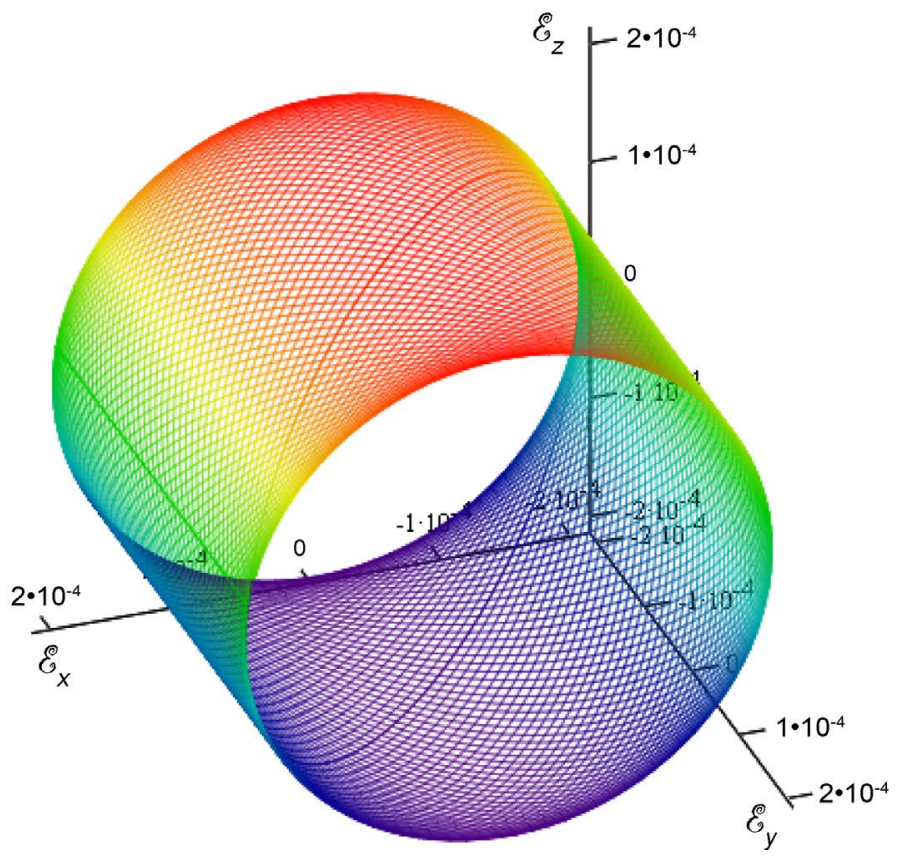

Figure 2. Cylindrical 3D polarization obtained with the configuration of Figure 1 with $\psi_{1}=\psi_{3}=0^{\circ}$ and $\psi_{2}$ varying continuously in the interval 0 to $2 \pi$.

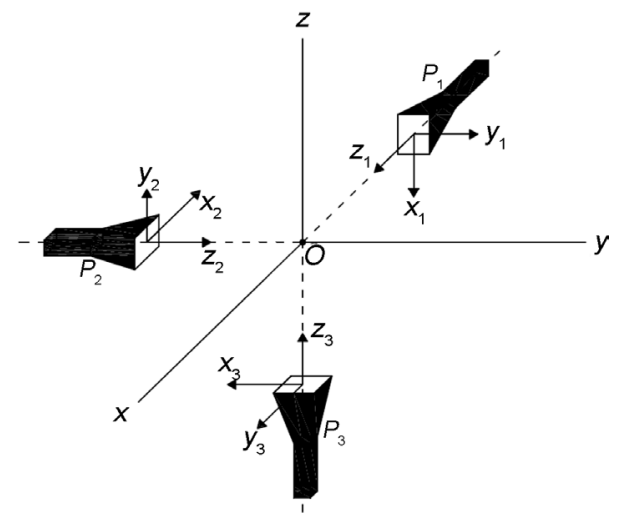

Figure 3. Array of pyramidal horn antennas.

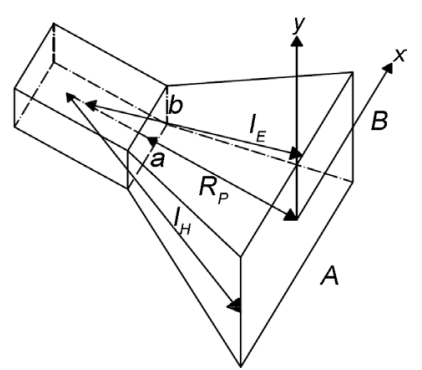

Figure 4. Geometry of a pyramidal horn antenna. 
The electric field of each horn can be approximated by (far-field) [19]

$$
\begin{aligned}
& \boldsymbol{E}_{\theta}=j \frac{k E_{0} \mathrm{e}^{-j k r}}{4 \pi r} \sin \phi(1+\cos \theta) I_{1} I_{2} \boldsymbol{a}_{\theta} \\
& \boldsymbol{E}_{\phi}=j \frac{k E_{0} \mathrm{e}^{-j k r}}{4 \pi r} \cos \phi(1+\cos \theta) I_{1} I_{2} \boldsymbol{a}_{\phi}
\end{aligned}
$$

where $E_{0}$ is the absolute value of the intensity of the electric field at the aperture (assuming the mode $\mathrm{TE}_{01}$ ). The electric field at the aperture is given by

$$
\boldsymbol{E}_{a y}=E_{0} \cos \left(\frac{\pi}{A} x\right) \boldsymbol{a}_{y}
$$

where $-A / 2 \leq x \leq A / 2$. The functions $I_{1}$ and $I_{2}$ are given by

$$
\begin{aligned}
I_{1}= & \frac{1}{2} \sqrt{\frac{\pi R_{1}}{k}}\left(\mathrm { e } ^ { j ( k _ { x } ^ { \prime 2 } R _ { 1 } / 2 k ) } \left\{\left[C\left(t_{2}^{\prime}\right)-C\left(t_{1}^{\prime}\right)\right]-j\left[S\left(t_{2}^{\prime}\right)-S\left(t_{1}^{\prime}\right)\right]\right.\right. \\
& \left.+\mathrm{e}^{j\left(k_{x}^{\prime \prime} R_{1} / 2 k\right)}\left\{\left[C\left(t_{2}^{\prime \prime}\right)-C\left(t_{1}^{\prime \prime}\right)\right]-j\left[S\left(t_{2}^{\prime \prime}\right)-S\left(t_{1}^{\prime \prime}\right)\right]\right\}\right) \\
I_{2}= & \sqrt{\frac{\pi R_{2}}{k}} \mathrm{e}^{j\left(k_{y}^{2} R_{2} / 2 k\right)}\left\{\left[C\left(t_{2}\right)-C\left(t_{1}\right)\right]-j\left[S\left(t_{2}\right)-S\left(t_{1}\right)\right]\right\}
\end{aligned}
$$

where $C(x)$ and $S(x)$ are the Fresnel functions, and $t_{1}^{\prime}, t_{2}^{\prime}, k_{x}^{\prime}, t_{1}^{\prime \prime}, t_{2}^{\prime \prime}, k_{x}^{\prime \prime}, t_{1}, t_{2}, k_{y}$ are given in [19]. Although the equations are not exact, the generation of 3D polarization from them can be demonstrated without loss of generality since a more exact approach would lead to different values in the radius of the sphere, which can always be compensated by adjusting properly the amplitude and/or phases of the element excitations (which in this example are the electric fields in the aperture of each antenna). Using electric fields distributions in the aperture of each horn as

$$
\begin{gathered}
\boldsymbol{E}_{1 a y}=E_{0} \sin (\zeta) \mathrm{e}^{-j \pi / 2} \cos \left(\frac{\pi}{A} x_{1}\right) \boldsymbol{a}_{y 1} \\
\boldsymbol{E}_{2 a y}=E_{0} \cos \left(\frac{\pi}{A} x_{2}\right) \boldsymbol{a}_{y 2} \\
\boldsymbol{E}_{3 a y}=E_{0} \cos (\zeta) \mathrm{e}^{-j \pi / 2} \cos \left(\frac{\pi}{A} X_{3}\right) \boldsymbol{a}_{y 3}
\end{gathered}
$$

where $\zeta$ is a variable controlled by the amplitude variation of the electric field in the aperture of each antenna, it can be shown that the total electrical field (far-field analysis) at the observation point $O$ is given by

$$
\boldsymbol{E}_{O}=C \cos (\zeta) \mathrm{e}^{-j \pi / 2} \boldsymbol{a}_{x}+C \sin (\zeta) \mathrm{e}^{-j \pi / 2} \boldsymbol{a}_{y}+C \boldsymbol{a}_{z}
$$

which in the time domain is

$$
\mathscr{E}_{0}(t)=C_{0}\left[\cos (\zeta) \sin \left(\omega t+\phi_{c}\right) \boldsymbol{a}_{x}+\sin (\zeta) \sin \left(\omega t+\phi_{c}\right) \boldsymbol{a}_{y}+\cos \left(\omega t+\phi_{c}\right) \boldsymbol{a}_{z}\right](10)
$$

where $C_{0}$ and $\varphi_{c}$ are respectively the absolute value and phase of (valid for $O$ at the origin)

$$
C=j \frac{k E_{0} \mathrm{e}^{-j k r}}{2 \pi r} I_{1}\left(\theta=0^{\circ}\right) I_{2}\left(\theta=0^{\circ}\right)
$$


The method introduced by the authors in [13] was employed to design each pyramidal horn (all equal to each other), and is herein omitted for simplicity. The optimum design ensures greater gain stability with respect to variations of the aperture dimensions [13]. The design gain was $18 \mathrm{dBi}$, frequency of $6 \mathrm{GHz}$ and the waveguide WR137 $\left(R_{1}=19.94 \mathrm{~cm}, R_{2}=18.2 \mathrm{~cm}, A=18.46 \mathrm{~cm}, B=\right.$ $14.19 \mathrm{~cm}$ ). The antennas are away $10^{3} \lambda$ from the origin (far-field), and $E_{0}=1$ $\mathrm{V} / \mathrm{m}$. The spherical polarization shown in Figure 5 is obtained by varying $\zeta$ continuously.

\section{Spatial Multiplexing by Electric Field Control}

In the previous sections the field control was done in only one point in the space; the reader is referred to [8] for a sensitivity analysis of degradations around that point. For spatial multiplexing, the fields should be controlled in many points in the space. This section shows the development of a method for controlling the electric field using three-dimensional array of antennas in multiple points in space, and how it can be applied in spatial multiplexing during the transmission of information in wireless digital communication systems.

The number of users in a given region can be increased with the new method, which is based on space division multiplexing, using the same frequency and time resources, with the least possible interference between users. Thus, the channel capacity can be increased. The interference can be further reduced between nearby users, increasing the signal to noise ratio $(\mathrm{S} / \mathrm{N})$ with the control of orthogonal polarizations.

\subsection{Method for Electric Field Control}

When $m$ antennas are used for the control of $n$ points in space, individual electric fields generated by each antenna are added to obtain the resulting field. The

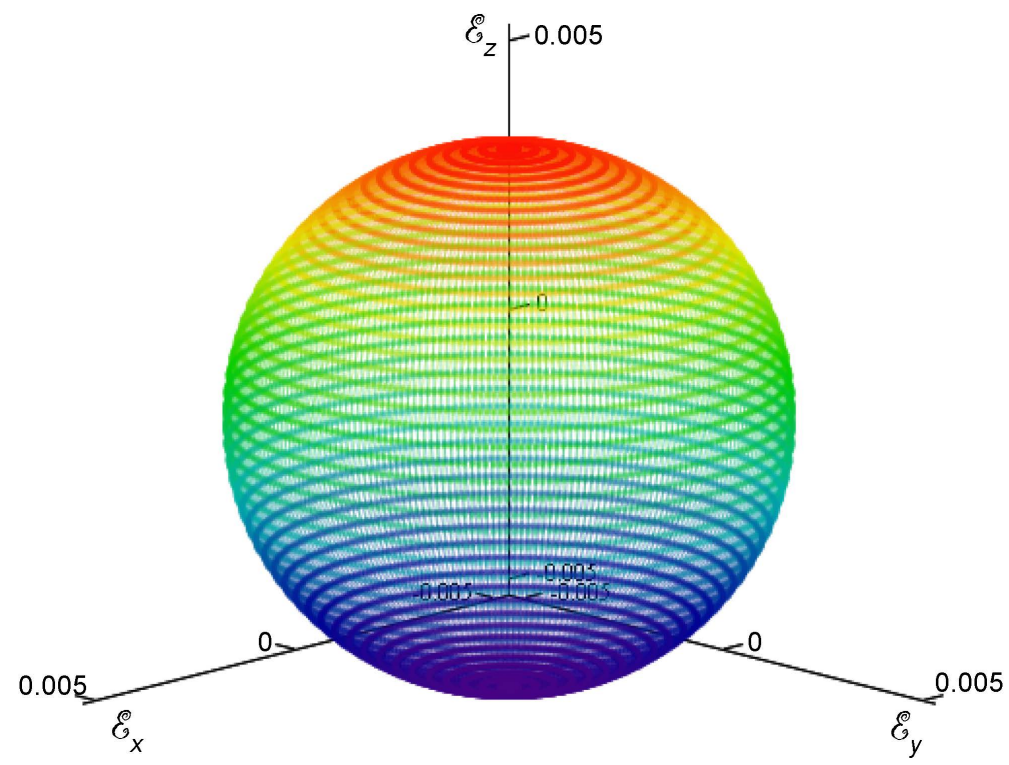

Figure 5. Spherical polarization with pyramidal horns (far-field). 
most direct method for this is to decompose the spherical components $\boldsymbol{a}_{r}$ and $\boldsymbol{a}_{\theta}$ directions in cartesian coordinates $\boldsymbol{a}_{x}, \boldsymbol{a}_{y}$ and $\boldsymbol{a}_{z}$ :

$$
\boldsymbol{E}=\left(E_{r} a_{r x}+E_{\theta} a_{\theta x}\right) \boldsymbol{a}_{x}+\left(E_{r} a_{r y}+E_{\theta} a_{\theta y}\right) \boldsymbol{a}_{y}+\left(E_{r} a_{r z}+E_{\theta} a_{\theta z}\right) \boldsymbol{a}_{z}
$$

where $a_{r x}, a_{r y}$ and $a_{r z}$ respectively represent the decomposition of the components of the unit vector $\boldsymbol{a}_{r}$ in the directions $\boldsymbol{a}_{x}, \boldsymbol{a}_{y}$ and $\boldsymbol{a}_{z}$. Also, $\boldsymbol{a}_{\theta x}, \boldsymbol{a}_{\theta y}$ and $\boldsymbol{a}_{\theta z}$ respectively represent the decomposition of the components of the unit vector $\boldsymbol{a}_{\theta}$ in the directions $\boldsymbol{a}_{x}, \boldsymbol{a}_{y}$ e $\boldsymbol{a}_{z}$.

Considering an antenna located at point $P_{1}$, and an observation point in $O_{1}$, the equations below are obtained in cartesian coordinates:

$$
\begin{gathered}
\boldsymbol{P}_{1}=x_{1} \boldsymbol{a}_{x}+y_{1} \boldsymbol{a}_{y}+z_{1} \boldsymbol{a}_{z}=\left(\begin{array}{lll}
x_{1} & y_{1} & z_{1}
\end{array}\right)^{\mathrm{T}} \\
\boldsymbol{S}_{1}=x_{s 1} \boldsymbol{a}_{x}+y_{s 1} \boldsymbol{a}_{y}+z_{s 1} \boldsymbol{a}_{z}=\left(\begin{array}{lll}
x_{s 1} & y_{s 1} & z_{s 1}
\end{array}\right)^{\mathrm{T}} \\
\boldsymbol{O}_{1}=x_{o 1} \boldsymbol{a}_{x}+y_{o 1} \boldsymbol{a}_{y}+z_{o 1} \boldsymbol{a}_{z}=\left(\begin{array}{lll}
x_{o 1} & y_{o 1} & z_{o 1}
\end{array}\right)^{\mathrm{T}}
\end{gathered}
$$

where $P_{1}$ is the position vector of antenna $1, S_{1}$ is the vector direction of antenna 1 , and $\boldsymbol{O}_{1}$ is the position vector of the observation point 1 . Superscript $\mathrm{T}$ in the equations was used for simplification, and indicates the transpose of the row vector. All these vectors are referenced to the origin of the cartesian system. $\boldsymbol{P}_{1}$ and $\boldsymbol{O}_{1}$ start from the origin and go to the specified points, and the distances represent the amplitudes. $S_{1}$ starts from point $\boldsymbol{P}_{1}$ and is defined here as a unit vector, and its amplitude equals 1 . The distance vector $D_{11}$ between $\boldsymbol{O}_{1}$ and $\boldsymbol{P}_{1}$ is $\boldsymbol{D}_{11}=\boldsymbol{O}_{1}-\boldsymbol{P}_{1}$. The angle formed between the vector $\boldsymbol{D}_{11}$ and $\boldsymbol{S}_{1}$ is given by

$$
\theta_{11}=\cos ^{-1}\left(\frac{\boldsymbol{D}_{11} \cdot \boldsymbol{S}_{1}}{\left|\boldsymbol{D}_{11}\right|}\right)
$$

The distance vector $D_{11}$ has the same direction of the vector $\boldsymbol{a}_{r 11}$. In general, establishing the variable $u$ as being equal to $x, y$ or $z, \boldsymbol{a}_{r u l 1}$ can be determined as:

$$
a_{r u 11}=\frac{D_{u 11}}{\left|\boldsymbol{D}_{11}\right|}
$$

With $\boldsymbol{a}_{r x 11}, \boldsymbol{a}_{r y 11}$, and $\boldsymbol{a}_{r z 11}$ values obtained through (17), $\boldsymbol{a}_{\theta x 11}, \boldsymbol{a}_{\theta y 11}$, and $\boldsymbol{a}_{\theta z 11}$ can be determined as:

$$
a_{\theta u 11}= \begin{cases}\frac{\left(\cos \theta_{11}\right) \boldsymbol{a}_{r 11}-\boldsymbol{S}_{1}}{\left|\cos \left(\theta_{11}\right) \boldsymbol{a}_{r 11}-\boldsymbol{S}_{1}\right|} \cdot \boldsymbol{a}_{u} & \text { if } \theta_{11} \neq 0 \\ 0 & \text { if } \theta_{11}=0\end{cases}
$$

Using $\boldsymbol{E}_{u 11}=\left(C_{u 11} \mathrm{e}^{j \varphi_{u 11}}\right)\left(I_{p 1} \mathrm{e}^{j \psi_{1}}\right) \boldsymbol{a}_{u}$, the contribution of the excitation source can be separated of the contribution responsible for the position and direction of the antenna:

$$
\begin{gathered}
C_{u 11}=\frac{\left|\boldsymbol{E}_{u 11}\right|}{I_{p 1}} \\
\mathrm{e}^{j \varphi_{u 11}}=\frac{\mathrm{e}^{j \varphi_{E u 11}}}{\mathrm{e}^{j \psi_{1}}}
\end{gathered}
$$


where $\varphi_{E u 11}$ is the argument of $\boldsymbol{E}_{u l 1}$. The coefficient $C_{u l 1}$ has the unit $\Omega / \mathrm{m} . C_{u l 1}$ and $\varphi_{E u 11}$ are calculated as

$$
\begin{gathered}
C_{u 11}=\frac{h}{4 \pi \omega \varepsilon r_{11}^{3}}\left\{\left(\alpha_{u 11}+\beta_{u 11}\right)^{2}+\left(k_{11} r_{11}\right)^{2}\left[\alpha_{u 11}^{2}+\left(\left(k_{11} r_{11}\right)^{2}-1\right) \beta_{u 11}^{2}\right]\right\}^{1 / 2} \\
\varphi_{u 11}=-k_{11} R_{11}+\operatorname{tg}^{-1}\left\{\frac{-\alpha_{u 11}+\beta_{u 11}\left[\left(k_{11} R_{11}\right)^{2}-1\right]}{k_{11} R_{11}\left(\alpha_{u 11}+\beta_{u 11}\right)}\right\}
\end{gathered}
$$

with

$$
\begin{gathered}
\alpha_{u 11}=2 \cos \theta_{11} a_{r u 11} \\
\beta_{u 11}=\sin \theta_{11} a_{\theta u 11}
\end{gathered}
$$

The phase and amplitude can be grouped in a single complex variable $\sigma_{u 11}$ as shown below

$$
\begin{gathered}
\sigma_{u 11}=C_{u 11} \mathrm{e}^{j \varphi_{u 11}} \\
\boldsymbol{E}_{u 11}=\sigma_{u 11}\left(I_{p 1} \mathrm{e}^{j \psi_{1}}\right) \boldsymbol{a}_{u}=\sigma_{u 11} I_{1} \boldsymbol{a}_{u}
\end{gathered}
$$

Once the values of the length of the antenna and the frequency of the excitation source are determined in the vacuum, the coefficient $\sigma_{u 11}$ will vary only with changes in the position and direction of the antenna, as well as the distance of this to the observation point. With $m$ antennas and $n$ observation points $(m=$ $3 n$ ), Equations (25) and (26) can be generalized to

$$
\begin{gathered}
\sigma_{u i l}=C_{u i l} \mathrm{e}^{j \varphi_{u i l}} \\
\boldsymbol{E}_{u i}=E_{u i} \boldsymbol{a}_{u}=\sum_{l=1}^{m} \boldsymbol{E}_{u i l}=\sum_{l=1}^{m} \sigma_{u i l} I_{l} \boldsymbol{a}_{u}=\sum_{l=1}^{m} \sigma_{u i l}\left(I_{p l} \mathrm{e}^{j \psi_{l}}\right) \boldsymbol{a}_{u}
\end{gathered}
$$

where $i$ and $l$ are integers number, with $1 \leq i \leq n, 1 \leq l \leq m$. Also, $u=x, y$ or $z$. Since $n$ can take any integer value, the electric field control in space can exist in the infinite points. In full matrix notation, this can be written as:

$$
\left(\begin{array}{cccc}
\sigma_{x 11} & \sigma_{x 12} & \cdots & \sigma_{x 1 m} \\
\sigma_{y 11} & \sigma_{y 12} & \cdots & \sigma_{y 1 m} \\
\sigma_{z 11} & \sigma_{z 12} & \cdots & \sigma_{z 1 m} \\
\sigma_{x 21} & \sigma_{x 22} & \cdots & \sigma_{x 2 m} \\
\sigma_{y 21} & \sigma_{y 22} & \cdots & \sigma_{y 2 m} \\
\sigma_{z 21} & \sigma_{z 22} & \cdots & \sigma_{z 2 m} \\
\vdots & \vdots & \ddots & \vdots \\
\sigma_{x n 1} & \sigma_{x n 2} & \cdots & \sigma_{x n m} \\
\sigma_{y n 1} & \sigma_{y n 2} & \cdots & \sigma_{y n m} \\
\sigma_{z n 1} & \sigma_{z n 2} & \cdots & \sigma_{z n m}
\end{array}\right)_{m \times m} \quad\left(\begin{array}{c}
I_{p 1} \mathrm{e}^{j \psi_{1}} \\
I_{p 2} \mathrm{e}^{j \psi_{2}} \\
\vdots \\
I_{p m} \mathrm{e}^{j \psi_{m}}
\end{array}\right)_{m \times 1}=\left(\begin{array}{c}
E_{x 1} \\
E_{y 1} \\
E_{z 1} \\
E_{x 2} \\
E_{y 2} \\
E_{z 2} \\
\vdots \\
E_{x n} \\
E_{y n} \\
E_{z n}
\end{array}\right)_{m \times 1}
$$

Calling $\sigma$ the matrix of coefficients, $I$ the current vector and $E$ the vector fields, the values of the excitation sources of the antennas can be determined by:

$$
\boldsymbol{I}=\boldsymbol{\sigma}^{-1} \boldsymbol{E}
$$


where $\sigma^{-1}$ is the inverse matrix of $\sigma$. In practice, numerical methods such as Gaussian elimination or LU decomposition are used because they are more efficient and accurate than the numerical computation of the inverse matrix [20]. Thus, the values of the amplitudes and phases of the current phasor are obtained with the desired values of $E$ at each point, as well with the calculation of the complex coefficient matrix $\sigma$.

\subsection{Digital Signal Transmission and Spatial Multiplexing Using the Method for Electric Field Control}

In this section the volumetric antennas array and fields control can be employed for the transmission of digital signals as well as to minimize the interference by spatial multiplexing. For the simulation, the antenna array presented in Figure 6 was used.

For the observation point $O_{1}$, the main antennas are 1, 2 and 3 because they contribute more significantly to the resulting electric field at this point, since the value of the coefficient $C_{u}$ has a higher value. Similarly, for point $O_{2}$, the main antennas are 4, 5 and 6. In the configuration of Figure 6, in each observation point the antennas are oriented such that the $\theta$ angle calculated by (26) is $90^{\circ}$.

Assuming $d_{O}=d_{A}=10 \lambda$, at an operating frequency of $100 \mathrm{MHz}$, the observation points are defined as $\boldsymbol{O}_{1}=\left(\begin{array}{llll}0 & -d_{O} & 0\end{array}\right)^{\mathrm{T}}$ and $\boldsymbol{O}_{2}=\left(\begin{array}{lll}0 & d_{O} & 0\end{array}\right)^{\mathrm{T}}$. The vectors positions and directions of the antennas are: $\boldsymbol{P}_{1}=\left(\begin{array}{lll}d_{A} & -d_{A} & 0\end{array}\right)^{\mathrm{T}}$, $\boldsymbol{P}_{2}=\left(\begin{array}{lll}0 & -2 d_{A} & 0\end{array}\right)^{\mathrm{T}}, \quad \boldsymbol{P}_{3}=\left(\begin{array}{lll}0 & -d_{A} & d_{A}\end{array}\right)^{\mathrm{T}}, \quad \boldsymbol{P}_{4}=\left(\begin{array}{lll}-d_{A} & d_{A} & 0\end{array}\right)^{\mathrm{T}}$, $\boldsymbol{P}_{5}=\left(\begin{array}{lll}0 & 2 d_{A} & 0\end{array}\right)^{\mathrm{T}}$ and $\boldsymbol{P}_{6}=\left(\begin{array}{llll}0 & d_{A} & -d_{A}\end{array}\right)^{\mathrm{T}} ; \boldsymbol{S}_{1}=\left(\begin{array}{lll}0 & 1 & 0\end{array}\right)^{\mathrm{T}}$, $\boldsymbol{S}_{2}=\left(\begin{array}{lll}0 & 0 & 1\end{array}\right)^{\mathrm{T}}, \quad \boldsymbol{S}_{3}=\left(\begin{array}{lll}1 & 0 & 0\end{array}\right)^{\mathrm{T}}, \quad \boldsymbol{S}_{4}=\left(\begin{array}{lll}0 & -1 & 0\end{array}\right)^{\mathrm{T}}, \quad \boldsymbol{S}_{5}=\left(\begin{array}{lll}0 & 0 & -1\end{array}\right)^{\mathrm{T}}$ and $\boldsymbol{S}_{6}=\left(\begin{array}{lll}-1 & 0 & 0\end{array}\right)^{\mathrm{T}}$.

Note that the mutual couplings between the transmitting antenna and the reception points are negligible, since the distances between the points of observations and the antennas are in far-fields. The amplitudes of the mutual impedances of the antennas in transmission and reception are considerably reduced if the distances between them are large [19]. Within this context, we obtain a matrix of complex coefficients $\sigma$.

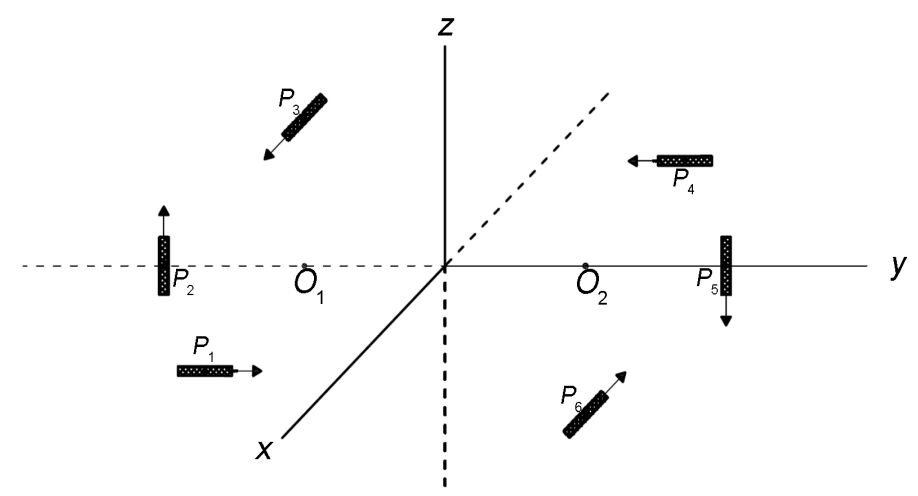

Figure 6. Array with 6 ideal antennas, working together for the electric field control of two observation points. 


$$
\sigma=\left(\begin{array}{cccccc}
0 & 0 & 0.628 \mathrm{e}^{j 4.696} & 0.112 \mathrm{e}^{-j 0.717} & 0 & 0.281 \mathrm{e}^{-j 0.703} \\
0.628 \mathrm{e}^{j 4.696} & 0 & 0 & 0.056 \mathrm{e}^{-j 0.646} & 0 & 0 \\
0 & 0.628 \mathrm{e}^{j 4.696} & 0 & 0 & 0.209 \mathrm{e}^{j 1.565} & 0 \\
0.112 \mathrm{e}^{j 2.425} & 0 & 0.281 \mathrm{e}^{j 2.439} & 0 & 0 & 0.628 \mathrm{e}^{j 1.555} \\
0.056 \mathrm{e}^{j 2.496} & 0 & 0 & 0.628 \mathrm{e}^{j 1.555} & 0 & 0 \\
0 & 0.209 \mathrm{e}^{j 4.707} & 0 & 0 & 0.628 \mathrm{e}^{j 1.555} & 0
\end{array}\right)_{6 \times 6}
$$

It was considered for the simulation digital transmissions ASK with binary signals with $m_{a}<1$. Although other modulations can be employed, we adopted this modulation for being simpler than the others, given that the focus of the simulation is to illustrate the transmission of signals to two different receiving points, sustaining isolation between the points at a same frequency and polarization.

For the simulation, it was also considered as bit 0 an amplitude of electric field equals to $2 \times 10^{-3} \mathrm{~V} / \mathrm{m}$, and bit 1 an amplitude equals to $4 \times 10^{-3} \mathrm{~V} / \mathrm{m}$. In the absence of transmission, the amplitude must be zero. A circular polarization was used in the plane $z=\frac{\sqrt{2}}{2} x+\frac{\sqrt{2}}{2} y$, noting that all antennas need to be excited to generate the desired electric field.

The simplest condition happens when there is no transmission at both observation points. In this configuration, the desired fields $E_{0}$ and $E_{1}$ are zero, and current feeds of the antennas are zero. For other conditions, Equation (30) was used to obtain the amplitudes and phases of the currents. Table 1 presents all results.

In the transition times of the desired signals, the currents must be changed in these moments. According to the table, all antennas must be fed, even when there is transmission at only one point. This is due to the fact that all antennas should work together to nullify the field in one of the reception points. It is also noted that the electric current amplitudes of main antennas for the point where

Table 1. Results of the antennas feed current for ASK transmission, achieving isolation between the two observations points.

\begin{tabular}{cccccccc}
\hline \multicolumn{7}{c}{ Desired Signal } & \multicolumn{5}{c}{ Antenna Current Phasors } \\
\hline $\boldsymbol{O}_{1}$ & $\boldsymbol{O}_{2}$ & $I_{1}(\mathrm{~mA})$ & $I_{2}(\mathrm{~mA})$ & $I_{3}(\mathrm{~mA})$ & $I_{4}(\mathrm{~mA})$ & $I_{5}(\mathrm{~mA})$ & $I_{6}(\mathrm{~mA})$ \\
\hline null & null & 0 & 0 & 0 & 0 & 0 & 0 \\
null & bit 0 & $0.246 \mathrm{e}^{j 3.49}$ & $0.844 \mathrm{e}^{-j 1.54}$ & $1.087 \mathrm{e}^{j 2.11}$ & $2.75 \mathrm{e}^{-j 0.59}$ & $2.532 \mathrm{e}^{-j 1.55}$ & $2.396 \mathrm{e}^{j 3.91}$ \\
null & bit 1 & $0.493 \mathrm{e}^{j 3.49}$ & $1.688 \mathrm{e}^{-j 1.54}$ & $2.173 \mathrm{e}^{j 2.11}$ & $5.5 \mathrm{e}^{-j 0.59}$ & $5.065 \mathrm{e}^{-j 1.55}$ & $4.792 \mathrm{e}^{j 3.91}$ \\
bit 0 & null & $2.75 \mathrm{e}^{j 2.55}$ & $2.532 \mathrm{e}^{j 1.59}$ & $2.396 \mathrm{e}^{j 0.76}$ & $0.246 \mathrm{e}^{j 0.35}$ & $0.844 \mathrm{e}^{j 1.6}$ & $1.087 \mathrm{e}^{-j 1.03}$ \\
bit 1 & null & $5.5 \mathrm{e}^{j 2.55}$ & $5.065 \mathrm{e}^{j 1.59}$ & $4.792 \mathrm{e}^{j 0.76}$ & $0.493 \mathrm{e}^{j 0.35}$ & $1.688 \mathrm{e}^{j 1.6}$ & $2.173 \mathrm{e}^{-j 1.03}$ \\
bit 0 & bit 0 & $2.902 \mathrm{e}^{j 2.62}$ & $1.688 \mathrm{e}^{j 1.58}$ & $2.845 \mathrm{e}^{j 1.15}$ & $2.902 \mathrm{e}^{-j 0.52}$ & $1.688 \mathrm{e}^{-j 1.56}$ & $2.845 \mathrm{e}^{j 4.29}$ \\
bit 0 & bit 1 & $3.066 \mathrm{e}^{j 2.68}$ & $0.844 \mathrm{e}^{j 1.57}$ & $3.579 \mathrm{e}^{j 1.39}$ & $5.649 \mathrm{e}^{-j 0.56}$ & $4.22 \mathrm{e}^{-j 1.56}$ & $5.147 \mathrm{e}^{j 4.11}$ \\
bit 1 & bit 0 & $5.649 \mathrm{e}^{j 2.58}$ & $4.221 \mathrm{e}^{j 1.59}$ & $5.147 \mathrm{e}^{j .9 .97}$ & $3.066 \mathrm{e}^{-j 0.46}$ & $0.844 \mathrm{e}^{j 4.71}$ & $3.579 \mathrm{e}^{j 4.54}$ \\
bit 1 & bit 1 & $5.804 \mathrm{e}^{j 2.62}$ & $3.377 \mathrm{e}^{j 1.58}$ & $5.69 \mathrm{e}^{j 1.15}$ & $5.804 \mathrm{e}^{-j 0.52}$ & $3.377 \mathrm{e}^{-j 1.56}$ & $5.69 \mathrm{e}^{j 4.29}$ \\
\hline
\end{tabular}


there is no transmission is small compared with the current amplitudes of the main antennas for the point where there is transmission.

Thus, all antennas work together and are responsible for the transmission of all signals. As each antenna carries a small portion of the information, the fading can be mitigated, due to the path diversity.

For illustration, Figure 7 shows the electric fields obtained when the desired signal is bit 1 at point 1 and null at point 2 .

Figure 7 shows that the electric fields were generated according to the desired signals, that is, a circular polarization in the plane $z=\frac{\sqrt{2}}{2} x+\frac{\sqrt{2}}{2} y$, with an amplitude equals to $4 \times 10^{-3} \mathrm{~V} / \mathrm{m}$ for the point of observation $O_{1}$ (a), and an amplitude equals zero for the point of observation $O_{2}(\mathrm{~b})$.

In this way, any electric field is generated at the observation point, regardless of the desired field at another point, resulting in a transmission isolated by spatial separation or spatial multiplexing.

To observe how the signal is degraded when users move away from the observation point used in the design, consider other example using the same conditions, except for $d_{O}=d_{A}=1000 \lambda$, which is approximately $3 \mathrm{~km}$ for $100 \mathrm{MHz}$. In order to obtain the same desired signals at $\boldsymbol{O}_{1}$ e $\boldsymbol{O}_{2}$, with the new current values obtained by (30), electric fields were simulated for different points $\boldsymbol{O}_{1 n}=\left(\begin{array}{lll}0 & -0.9 d_{O} & 0\end{array}\right)^{\mathrm{T}}$ and $\boldsymbol{O}_{2 n}=\left(\begin{array}{lll}0 & 0.9 d_{O} & 0\end{array}\right)^{\mathrm{T}}$.

The time-average amplitude of the resulting field at the observation point $i$ is calculated as follows:

$$
\mathscr{E}_{a i}=\frac{1}{T_{b}} \int_{0}^{T_{b}} \sqrt{\mathscr{E}_{x i}^{2}(t)+\mathscr{E}_{y i}^{2}(t)+\mathscr{E}_{z i}^{2}(t)} \mathrm{d} t
$$

In possession of the $\mathscr{E}_{a i}$ value, the error of time-average amplitude of the resulting field regarding the value of the desired field $\mathscr{E}_{d i}$ is calculated as:

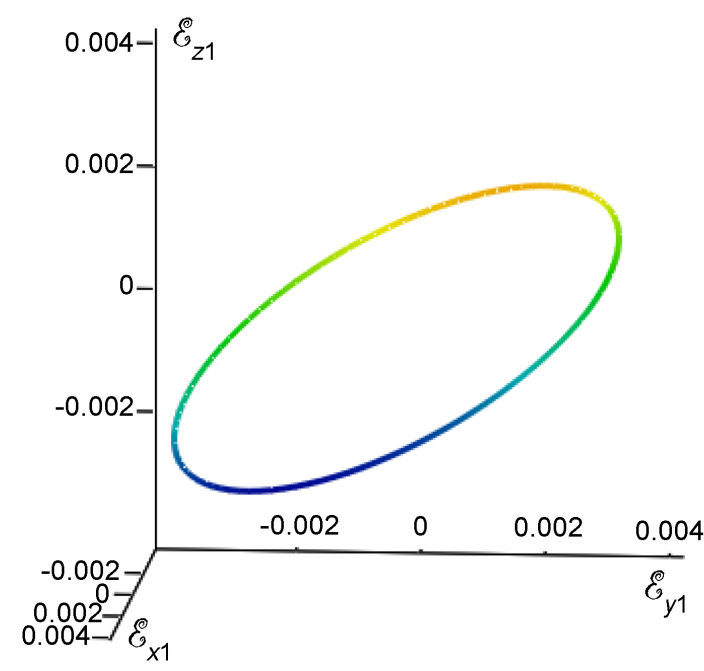

(a)

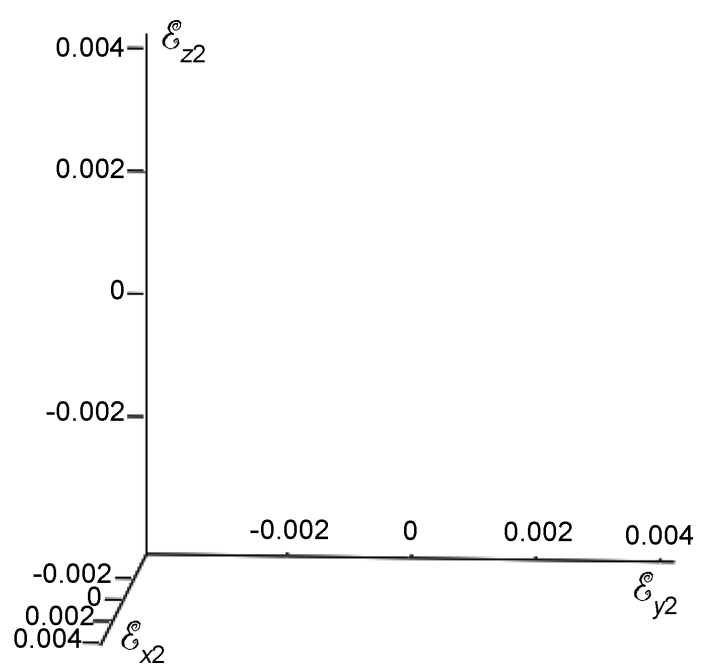

(b)

Figure 7. Instantaneous electric fields resulting in points of observations $O_{1}$ (a) and $O_{2}$ (b), given in V/m, when the desired signals are bit 1 at $O_{1}$ and null at $O_{2}$, and the antenna feed current shown in Table 1 . 


$$
\Delta \mathscr{E}_{i}=\mathscr{E}_{a i}-\mathscr{E}_{d i}
$$

Instead of calculating the difference of electric field amplitude at each instant of time, (32) was used in order not to consider the propagation delays between observations points. As the polarizations are in different planes, and the observations points are separated by multiple wavelengths, the Equation (32) becomes more appropriate to the intended results. Table 2 shows these results.

In Table 2, the positive or negative sign of the value of the error indicates that the time-average amplitude of the field is increased or reduced. It is evident that, when the desired signals are not zero at the points of observations, the errors are minor compared to the lack of transmission. In both cases, the errors are small, though the observations points used in the design are about $300 \mathrm{~m}$ away from the receptions points. Figure 8 shows the resulting electric fields for one case.

Despite that the $O_{1 n}$ and $O_{2 n}$ points are approximately $300 \mathrm{~m}$ from the points $\boldsymbol{O}_{1}$

Table 2. Errors of time-average amplitude of the resulting electric field for the $O_{1 n}$ and $\mathrm{O}_{2 n}$ points.

\begin{tabular}{|c|c|c|c|c|c|c|c|}
\hline \multicolumn{2}{|c|}{ Desired Signal } & \multicolumn{4}{|c|}{ Time-average amplitude of the resulting field } & \multicolumn{2}{|c|}{ Error } \\
\hline$O_{1}$ & $\mathrm{O}_{2}$ & $\mathscr{E}_{d 1}(\mathrm{~V} / \mathrm{m})$ & $\mathscr{E}_{d 2}(\mathrm{~V} / \mathrm{m})$ & $\mathscr{E}_{a 1}(\mathrm{~V} / \mathrm{m})$ & $\mathscr{E}_{a 2}(\mathrm{~V} / \mathrm{m})$ & $\Delta \mathscr{E}_{1}(\mathrm{~V} / \mathrm{m})$ & $\Delta \mathscr{E}_{2} \quad(\mathrm{~V} / \mathrm{m})$ \\
\hline null & bit 0 & 0 & $2 \times 10^{-3}$ & $0.143 \times 10^{-3}$ & $1.932 \times 10^{-3}$ & $0.143 \times 10^{-3}$ & $-0.068 \times 10^{-3}$ \\
\hline null & bit 1 & 0 & $4 \times 10^{-3}$ & $0.286 \times 10^{-3}$ & $3.865 \times 10^{-3}$ & $0.286 \times 10^{-3}$ & $-0.135 \times 10^{-3}$ \\
\hline bit 0 & null & $2 \times 10^{-3}$ & 0 & $1.932 \times 10^{-3}$ & $0.143 \times 10^{-3}$ & $-0.068 \times 10^{-3}$ & $0.143 \times 10^{-3}$ \\
\hline bit 1 & null & $4 \times 10^{-3}$ & 0 & $3.865 \times 10^{-3}$ & $0.286 \times 10^{-3}$ & $-0.135 \times 10^{-3}$ & $0.286 \times 10^{-3}$ \\
\hline bit 0 & bit 0 & $2 \times 10^{-3}$ & $2 \times 10^{-3}$ & $1.942 \times 10^{-3}$ & $1.942 \times 10^{-3}$ & $-0.058 \times 10^{-3}$ & $-0.058 \times 10^{-3}$ \\
\hline bit 0 & bit 1 & $2 \times 10^{-3}$ & $4 \times 10^{-3}$ & $1.962 \times 10^{-3}$ & $3.871 \times 10^{-3}$ & $-0.038 \times 10^{-3}$ & $-0.129 \times 10^{-3}$ \\
\hline bit 1 & bit 0 & $4 \times 10^{-3}$ & $2 \times 10^{-3}$ & $3.871 \times 10^{-3}$ & $1.962 \times 10^{-3}$ & $-0.129 \times 10^{-3}$ & $-0.038 \times 10^{-3}$ \\
\hline bit 1 & bit 1 & $4 \times 10^{-3}$ & $4 \times 10^{-3}$ & $3.883 \times 10^{-3}$ & $3.883 \times 10^{-3}$ & $-0.117 \times 10^{-3}$ & $-0.117 \times 10^{-3}$ \\
\hline
\end{tabular}

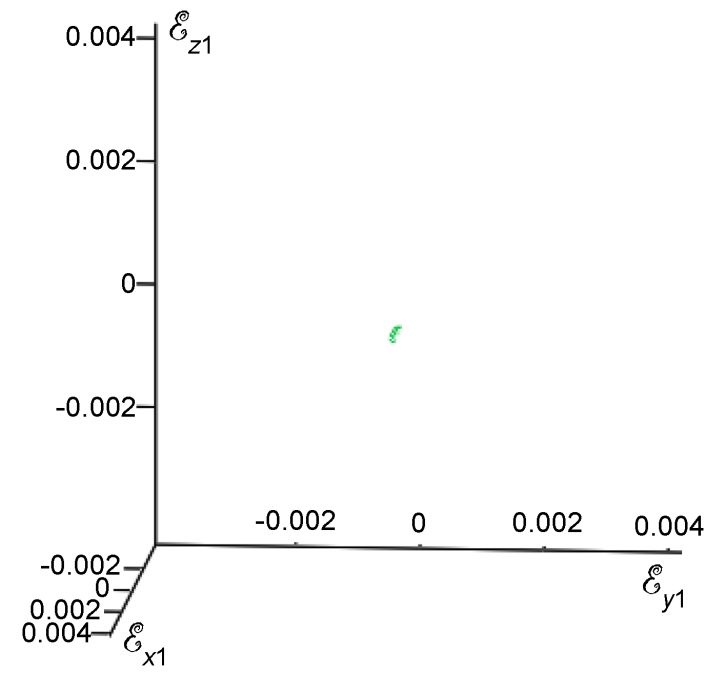

(a)

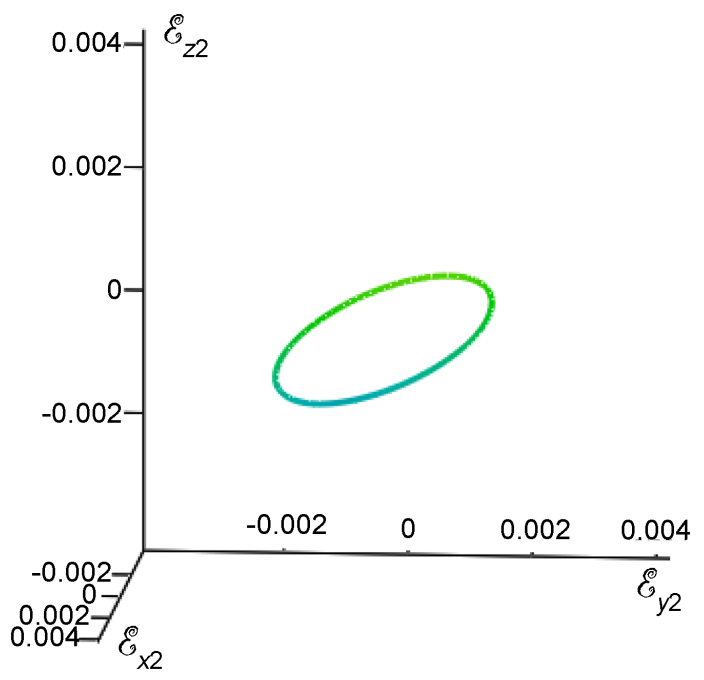

(b)

Figure 8. Instantaneous electric fields resulting at points of observations $O_{1 n}$ (a) and $O_{2 n}(\mathrm{~b})$, given in $\mathrm{V} / \mathrm{m}$, when the desired signals are null at $O_{1}$ and bit 0 at $O_{2}$. 
and $O_{2}$, the amplitudes of the electric fields generated and the polarization planes almost did not suffer degradation.

Considering the small decoupling between the receiving antennas due to the difference between the planes of polarization, the received power will be slightly smaller. Consequently, the largest error obtained in the example will have a small reduction.

In fact, spatial regions whose deviations are small are formed around the observation point $\boldsymbol{O}_{i}$, and the isolation is preserved. Thus, the reception point could move within that region without suffer interference. The region depends on the threshold detection to identify the signals.

Although the distance between the new observations points and those used in the design are large, it represents only a percentage of the distance from the observation points $\boldsymbol{O}_{1}$ and $\boldsymbol{O}_{2}$ to the origin, which equals $3.000 \mathrm{~m}$. Thus, as these points move away from their main antennas, the greater will be the region of reception, where users can move without any significant errors. This is because the amplitude of the electric fields suffers smooth transitions between the two observation points.

Ignoring the effects of fading, the theoretical perfect isolation can be maintained if the system knows the locations of all the reception points. So with the new position of the observation point, the matrix of complex coefficients $\sigma$ could be updated, if necessary and new values of currents are obtained using (30). The sensitivity of the signals with respect to various distances from the target points, as well as case examples with pyramidal horn antennas, is discussed in depth in [21]. Furthermore, this approach can be of substantial interest when considering the fact that cellular systems are moving towards micro and phantom cells [14].

\section{Torus-Knots Polarizations and Fields}

The theory and possible applications related to torus-knotted electromagnetic fields is discussed in [16] [17], which can be encountered in nature around celestial objects and are used for plasma confinement and with nuclear magnetic resonance devices. Here we only comment for completeness that antenna array configurations similar to the ones presented in the previous sections can also be employed to generate these distributions in a controlled and consistent fashion. Furthermore, we show that not only the torus-knotted fields but polarizations as well can be obtained with the procedure [21]. Figure 9 and Figure 10 show an example of torus-knots for an electromagnetic three-dimensional polarization distribution (as time varies). The synthesis procedure is herein omitted for simplicity, as well as the torus-knotted field distributions (at a given instant of time), and the reader is referred to [21] for more information.

\section{Conclusions}

This work extended substantially a procedure introduced by the authors in [8] to generate three-dimensional (3D) polarizations, where the electric field intensity 


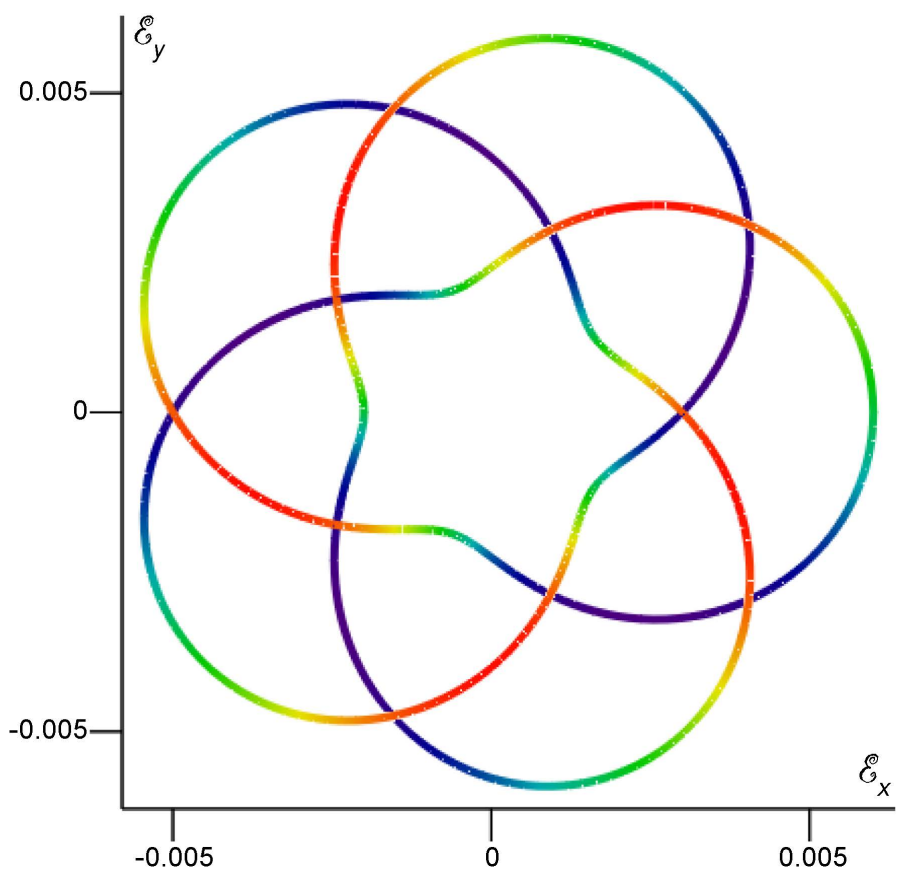

Figure 9. An illustrative example of torus-knotted polarization in the $x y$-plane, produced by an array of 3 ideal dipole antennas. Although not herein shown similar distributions for the electromagnetic fields at a given instant of time can also be obtained [21].

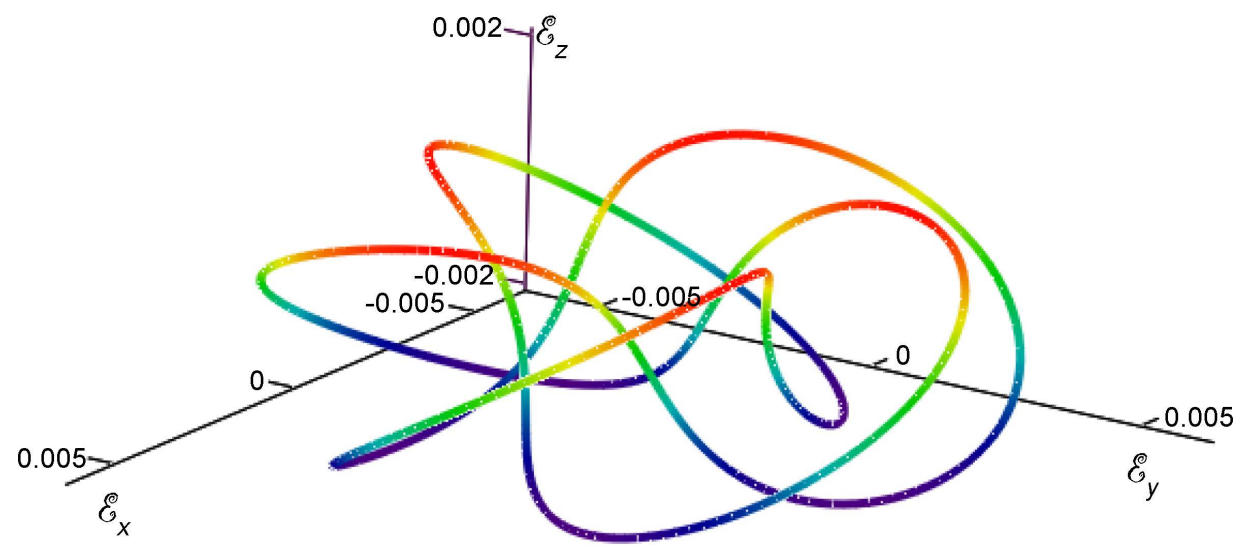

Figure 10. An illustrative example of torus-knotted polarization (three-dimensional view of Figure 9). Although not herein shown similar distributions for the electromagnetic fields at a given instant of time can also be obtained [21].

vector can be oriented in any direction in space. These polarizations were referred to as "3D" due to their distribution in space, although time is also inherent. It was shown analytically and numerically that it is possible to generate $3 \mathrm{D}$ polarizations with different shapes, as time varies, such as cylinders, spheres, and others. In contrast to [8] where only ideal dipoles were employed for generating $3 \mathrm{D}$ polarizations, here pyramidal horns were also used, not only for polarizations but also to generate controlled electromagnetic field distributions. In addition, the orthogonality between 3D polarizations was also mentioned. It is worth noting that for the scenarios considered in this study, there is no propagation, so 
the $3 \mathrm{D}$ polarization needs to be visualized as the polarization of the resulting electric field, not the wave. Alternate configurations that allow the $3 \mathrm{D}$ polarization to move within a desired path are currently under investigation (by determining different sets of feed currents for each desired location, for example), but the basic theory as presented here does not lose its generality. In fact, more accurate results can be obtained with the numerical modeling using commercial and/or in-house antenna codes, which will also allow determining the correct excitations to compensate for effects not accounted here, such as mutual coupling in smaller cells, near-field effects for non-ideal antennas, reflections, refractions, etc...

Although the main purpose was to introduce a mathematically consistent synthesis procedure for controlling the three-dimensionally distributed electromagnetic fields and polarizations, with applications yet to be adapted or discovered, an effort was made to illustrate that the technique can be used for digital transmission of information in wireless communication systems with spatial multiplexing. For each observation point, three antennas will be necessary. If more antennas are used, the fading will be smaller and the results will be closer to the desired. Volumetric regions are created with this array, and isolation could be preserved if the reception points are within these regions. The interference for spatial multiplexing can be further reduced if the method of electric field control is combined with the use of orthogonal 3D polarizations between close users, as in nano and phantom cellular systems [14]. Finally, another possible use of the theory was briefly mentioned for torus-knotted electromagnetic fields, as well as illustrated for three-dimensional polarization distributions. Torus-knotted fields can be found naturally around various celestial objects, as historically shown in Radioastronomy as well as possibly to be further investigated with advanced Radiotelescopes [15], and can be of importance for plasma confinement and nuclear magnetic resonance devices [16] [17]. Measurement procedures for three-dimensional electromagnetic fields in space (at a given instant of time) and polarizations (as time varies) are subject of further discussion, as for mathematical multi-dimensional results.

\section{References}

[1] Stutzman, W.L. and Thiele, G.A. (2012) Antenna Theory and Design. 3rd Edition, John Wiley \& Sons, Hoboken.

[2] Paul, C.A. (2006) Introduction to Electromagnetic Compatibility. 2nd Edition, John Wiley \& Sons, Hoboken.

[3] Terada, M. (2005) Reflector Antennas. In: Chang, K., Ed., Wiley Encyclopedia of RF and Microwave Engineering, 5, 4450-4474.

[4] Forenza, A. and Heath, R.W. (2006) Benefit of Pattern Diversity via Two-Element Array of Circular Patch Antennas in Indoor Clustered MIMO Channels. IEEE Transactions on Communications, 54, 943-954. https://doi.org/10.1109/TCOMM.2006.873978

[5] Zooghby, A.E. (2005) Smart Antenna Engineering. Artech House, London. 
[6] Stutzman, W.L. (1992) Polarization in Electromagnetic Systems. Artech House, London.

[7] Terada, M.A.B. (2003) Novel Beam Scanning Technique for Circularly Polarized Reflector Antennas. Proceedings of the IEEE International Symposium on Antennas and Propagation, Columbus, 22-27 June 2003, 770-773.

https://doi.org/10.1109/aps.2003.1219349

[8] Pereira, L.P.S. and Terada, M.A.B. (2012) Three-Dimensional Electromagnetic Polarization. Brazilian Symposium of Microwaves and Optoelectronics (MOMAG 2012), 1, 1-5.

[9] Terada, M.A.B. (2004) Distributed Three-Dimensional Array Configuration for Volumetric-Controlled Field Coverage: A New Concept for High-Efficiency, Distance-Selective Wireless Local Area Network. URSI-B Symposium on Electromagnetic Theory (URSI_EMTS), Pisa, 23-27 May 2004, 975-977.

[10] Terada, M.A.B. (2004) Distance-Selective Wireless Network with Enhanced Isolation Characteristics: A New Concept Based on Three-Dimensional Wave Synthesis for Volumetric-Controlled Field Coverage. Proceedings of the 2004 IEEE International Symposium on Antennas and Propagation, Monterey, 20-25 June 2004, 2, 1736-1739. https://doi.org/10.1109/APS.2004.1330532

[11] Piestun, R. and Shamir, J. (2002) Synthesis of Three-Dimensional Light Fields and Applications. Proceedings of the IEEE, 90, 222-244.

[12] Berkhout, A.J. (1988) A Holographic Approach to Acoustic Control. Journal of the Audio Engineering Society, 36, 977-995.

[13] Pereira L.P.S. and Terada, M.A.B. (2011) New Method for Optimum Design of Pyramidal Horn Antennas. Journal of Microwaves, Optoelectronics and Electromagnetic Applications, 10, 266-277. https://doi.org/10.1590/S2179-10742011000100025

[14] Wanderley, P. and Terada, M. (2012) Assessment of the Applicability of the Ikegami Propagation Model in Modern Wireless Communication Scenarios. Journal of Electromagnetic Waves and Applications, 26, 1483-1491. https://doi.org/10.1080/09205071.2012.703077

[15] Young, André, Terada, Marco A.B., de Villiers, Dirk I.L. and Davidson, David B. (2012) Assessment of the Sensitivity of the South African KAT-7 and MeerKAT/SKA Radio Telescope Reflector Antennas. IEEE 2012 International Conference on Electromagnetics in Advanced Applications, 1, 486-489. https://doi.org/10.1109/ICEAA.2012.6328676

[16] Arrayás, M. and Trueba, J.L. (2015) A Class of Non-Null Toroidal Electromagnetic Fields and Its Relation to the Model of Electromagnetic Knots. Journal of Physics A: Mathematical and Theoretical, 48, 1-14. https://doi.org/10.1088/1751-8113/48/2/025203

[17] Irvine, W.T.M. and Bouwmeester, D. (2008) Linked and Knotted Beams of Light. Nature Physics, 4, 716-720. https://doi.org/10.1038/nphys1056

[18] Mathcad (2017) www.ptc.com/engineering-math-software/mathcad

[19] Balanis, C.A. (2005) Antenna Theory: Analysis and Design. 3rd Edition, John Wiley $\&$ Sons, Hoboken.

[20] Chapra, S.C. and Canale, R.P. (2009) Numerical Methods for Engineers. 6th Edition, McGraw Hill Science/Engineering/Math, New York.

[21] Pereira, L.P.S. (2015) Synthesis of Antennas for Field and Polarization Control. Ph.D. Dissertation, University of Brasilia, Brasilia. 
Submit or recommend next manuscript to SCIRP and we will provide best service for you:

Accepting pre-submission inquiries through Email, Facebook, LinkedIn, Twitter, etc. A wide selection of journals (inclusive of 9 subjects, more than 200 journals)

Providing 24-hour high-quality service

User-friendly online submission system

Fair and swift peer-review system

Efficient typesetting and proofreading procedure

Display of the result of downloads and visits, as well as the number of cited articles Maximum dissemination of your research work

Submit your manuscript at: http://papersubmission.scirp.org/

Or contact jemaa@scirp.org 